\title{
Scoping review of complexity theory in health services research
}

\author{
David S. Thompson ${ }^{1 *}$, Xavier Fazio ${ }^{2}$, Erika Kustra ${ }^{3}$, Linda Patrick ${ }^{4}$ and Darren Stanley ${ }^{5}$
}

\begin{abstract}
Background: There are calls for better application of theory in health services research. Research exploring knowledge translation and interprofessional collaboration are two examples, and in both areas, complexity theory has been identified as potentially useful. However, how best to conceptualize and operationalize complexity theory in health services research is uncertain. The purpose of this scoping review was to explore how complexity theory has been incorporated in health services research focused on allied health, medicine, and nursing in order to offer guidance for future application. Given the extensiveness of how complexity theory could be conceptualized and ultimately operationalized within health services research, a scoping review of complexity theory in health services research is warranted.

Methods: A scoping review of published research in English was conducted using CINAHL, EMBASE, Medline, Cochrane, and Web of Science databases. We searched terms synonymous with complexity theory.

Results: We included 44 studies in this review: 27 were qualitative, 14 were quantitative, and 3 were mixed methods. Case study was the most common method. Long-term care was the most studied setting. The majority of research was exploratory and focused on relationships between health care workers. Authors most commonly used complexity theory as a conceptual framework for their study. Authors described complexity theory in their research in a variety of ways. The most common attributes of complexity theory used in health services research included relationships, self-organization, and diversity. A common theme across descriptions of complexity theory is that authors incorporate aspects of the theory related to how diverse relationships and communication between individuals in a system can influence change.

Conclusion: Complexity theory is incorporated in many ways across a variety of research designs to explore a multitude of phenomena.. Although complexity theory shows promise in health services research, particularly related to relationships and interactions, conceptual confusion and inconsistent application hinders the operationalization of this potentially important perspective. Generalizability from studies that incorporate complexity theory is, therefore, difficult. Heterogeneous conceptualization and operationalization of complexity theory in health services research suggests there is no universally agreed upon approach of how to use this theory in health services research. Future research should include clear definitions and descriptions of complexity and how it was used in studies. Clear reporting will aid in determining how best to use complexity theory in health services research.
\end{abstract}

Keywords: Complexity theory, Health services research, Theory

\footnotetext{
* Correspondence: dsthomp1@lakeheadu.ca

${ }^{1}$ School of Nursing, Lakehead University, 955 Oliver Road, Thunder Bay, ON

P7B 5E1, Canada

Full list of author information is available at the end of the article
} 


\section{Background}

There are calls to increase the use of theory when designing and conducting health services research. Knowledge translation and interprofessional collaboration are two areas of health services research experiencing such calls. Knowledge translation research is the study of how best to ensure stakeholders are made aware of, and use, research evidence in decision-making [1]. Interprofessional collaboration research explores how best to support professionals to develop and maintain optimal working relationships [2]. Together, knowledge translation and interprofessional collaboration research hold potential for improving health care processes and outcomes [3], nonetheless they share a common criticism. Researchers report low numbers of studies where authors have used theory in their research $[4,5]$ and such reports have prompted calls for improvement.

Theory is important in designing and conducting both qualitative and quantitative research on phenomena related to health services (e.g., knowledge translation, interprofessional collaboration) as it aids in the development of generalizable and robust knowledge [6, 7]. Explicit use of theory can assist a reader to decide whether findings are applicable and useable in specific settings. Overviews identifying potentially useful theories exist in both knowledge translation and interprofessional collaboration [7, 8]. Authors in both fields suggest that considering theoretical perspectives that include attributes of complexity theory may be useful in a study's design and data analysis [7, 9-11].

\section{Complexity theory}

Definitions of complexity theory are elusive and "there is no generally accepted statement of what complexity theory is or how complex something must be to come with the ambit of complexity theory" [12]. Conceptual confusion associated with complexity theory may reflect questionable validity, transdisciplinarity [13], and/or lack of in depth knowledge by researchers of the methodological considerations for complexity theory. However, the absence of a universal definition is not akin to an absence of validity. For instance, the transdisciplinary nature of complexity theory is a plausible explanation for an elusive definition because "any definition of complexity is beholden to the perspective brought to bear upon it" [14]. Definitions of complexity are often tailored to reflect the phenomena of interest [15]. Despite authors using complexity theory, little is known on how to conceptualize and operationalize this theory to best suit health services research. For the purpose of this review, we align ourselves with Cilliers' [16] description of complexity theory: "complexity is a characteristic of a system". Specifically, for this review, we view complexity theory as a perspective that conceptualizes relationships of components (i.e., individuals) within a system as the foundation from which the properties of a system emerge.

Drawing from Cilliers [16], and Strumberg and Martin's [17] work, we offer some propositions of complexity theory. First, complexity theory offers a perspective to studying complex systems in a manner that does not reduce the system to individual components. From a complexity theory perspective, the interactions between components of a system are important for studying a system. Second, it is the interactions of system components that result in the overall behavior of the system. Complexity theory acknowledges that agents within a system interact to produce such behavior. Using complexity language, self-organization refers to the interactions between agents and emergence refers to the system level changes. Third, the interactions between agents are not controlled by a central control. Interactions arise from individual agents following simple rules and responding to environmental changes-control is decentralized. Fourth, the system is open to the surroundings. Interaction of the agents with their surroundings results in the exchange of information and people. These exchanges influence how those agents interact. Finally, agents have limited control over how system level changes emerge. As such, new system behavior is often unpredictable and difficult to trace back to a specific cause. These propositions, while not exhaustive, offer a general understanding of complexity theory for the purposes of our review.

Reviews of complexity theory exist in organizational science [18], mathematics and management [19], and health care [20]. Wallis [18] examined how complexity was used in the organizational science literature and concluded there was great diversity in application. In turn, he called for a more explicit and comprehensive application of the concepts of complexity. Pollack et al. [19] compared the use of complexity theory between mathematics and organizational science research. They found researchers in organizational science, although late adopters of complexity theory when compared to researchers in mathematics, are continuing to explore ways of applying complexity theory to management questions. These findings were consistent with a review by Sturmberg et al. [20] exploring the evolution of family medicine/general practice from a complex systems perspective. Like Pollack et al [19], Sturmberg et al. [20] found researchers were applying complexity theory more frequently than several decades ago. Notwithstanding, social science researchers use complexity in a metaphorical manner whereas computer science and mathematics use complexity for quantitative modeling. Across all three reviews, conclusions suggested that the "proper" or "feasible" application of complexity to social contexts remains unknown. 
Researchers are increasingly incorporating complexity theory in health services research despite ongoing debate on how best to do it [21-23]. There are no reviews exploring how complexity theory has been incorporated in the broader health services research literature related to nursing, medicine, and allied health. Given the extensiveness of how complexity theory could be conceptualized and ultimately operationalized within health services research, a scoping review of complexity theory in health services research is warranted.

The purpose of this scoping review is to explore how complexity theory has been incorporated in health services research. In doing so, we answer the following research questions:

1. What are the characteristics of studies that use complexity theory in health services research?

2. What settings and professions do researchers study using complexity theory?

3. What research questions and phenomena of interest do researchers focus on when using complexity theory?

4. How are researchers using complexity theory within health services research ${ }^{1}$ ?

5. How are researchers describing complexity theory within health services research?

\section{Methods}

We anticipated heterogeneous studies in terms of research purposes, phenomena of interest, methods, participants, and context. Likewise, although we aimed to conduct a broad, replicable, and systematic search of published literature, we did not seek to appraise and synthesize research evidence. Therefore, a systematic review was not warranted. In an evaluation of review methods, Grant and Booth [24] described scoping reviews as "a preliminary assessment of potential size and scope of available research literature". Arksey and O'Malley [25] and Levac, Colquhoun, and O'Brien [26] have developed and advanced the recommended methodological framework for scoping reviews [27]. Scoping reviews involve five steps: (a) identifying the initial research question; (b) identifying the relevant studies; (c) selecting the studies; (d) charting the results; (e) collating, summarizing, and reporting the findings; and (f) consulting stakeholders for knowledge translation of findings [25]. With the exception of consultation of stakeholders, we followed Arksey and O'Malley's approach, and used Levac et al. as a guide, for how to operationalize each step.

\section{Identifying relevant studies}

Literature published between inception of each database and June 2015 was collected from the following databases: The Cochrane Database of Systematic Reviews, CINAHL, EMBASE, Medline, and Web of Science. The search strategy and database selection was determined in consultation with a Master of Library Information Science (MLIS) Librarian and a researcher familiar with complexity theory (DS). Table 1 outlines the search strategy for each database. Given the breadth of complexity theory, combined with a lack of agreed upon nomenclature, we anticipated literature to be indexed under a variety of terms. To account for broad indexing, we used a range of search terms often associated with complexity theory. We used citation searching when key articles were found.

\section{Study selection}

A study was eligible for inclusion if: (a) it was published in a peer-reviewed journal, (b) it was written in English, (c) authors provided a statement somewhere in their manuscript reporting they incorporated complexity theory within their research, (d) authors studied a phenomena related to health services research, and (e) authors included nurses, physicians, or allied health professionals.

For criterion $c$ we did not exclude studies on the basis of study design.

Articles describing quality improvement projects were excluded, but articles describing quality improvement research or research on quality improvement techniques were included. We excluded articles describing quality improvement projects because the focus of quality improvement projects differs from that of research, with the former focused on descriptions of how a group worked to improve care for a specific population or

Table 1 Search strategy by database

\begin{tabular}{|c|c|}
\hline Database & Search strategy \\
\hline CINAHL & $\begin{array}{l}\text { Complexity theory OR complexity science OR } \\
\text { complex adaptive system OR complexity } \\
\text { thinking OR complex responsive process } \\
\text { theory OR chaos theory }\end{array}$ \\
\hline $\begin{array}{l}\text { Cochrane Database of } \\
\text { Systematic Reviews }\end{array}$ & $\begin{array}{l}\text { Complexity theory OR complexity science OR } \\
\text { complex adaptive system OR complexity } \\
\text { thinking OR chaos theory OR complex } \\
\text { responsive process theory }\end{array}$ \\
\hline EMBASE & $\begin{array}{l}\text { Complexity theory OR complexity science OR } \\
\text { complex adaptive system OR complexity } \\
\text { thinking OR chaos theory OR complex } \\
\text { responsive process theory }\end{array}$ \\
\hline Medline & $\begin{array}{l}\text { Complexity theory OR complexity science OR } \\
\text { complex adaptive system OR complexity } \\
\text { thinking OR chaos theory OR complex } \\
\text { responsive process theory }\end{array}$ \\
\hline Web of science & $\begin{array}{l}\text { TS = ("complexity theory" OR "complexity } \\
\text { science" OR "complex adaptive system" OR } \\
\text { "complexity thinking" OR "complex responsive } \\
\text { process theory" OR "chaos theory") DocType = All } \\
\text { document types; Language = All languages }\end{array}$ \\
\hline
\end{tabular}


organization and the later focused on developing new and (often) generalizable knowledge [28]. Our focus is on complexity theory in health services research, thus we excluded descriptions of quality improvement projects. Distinguishing between quality improvement and research reports is difficult [29]. To assist, we used criteria described by Newhouse et al. [28] that included assessment of intent of the authors, burdens and risks to subjects, and oversight of the project.

For criterion $d$, we used the Canadian Institute of Health Research definition of health services research [30]. We excluded studies that used complexity theory to explain aspects of diseases (e.g., atrial fibrillation, cerebral vascular accidents). Likewise, we excluded studies offering commentary or discussion articles on how complexity theory could be used in research.

For criterion $e$ we defined allied health professionals as dietitians, occupational therapists, pharmacists, physiotherapists, and speech-language pathologists. If studies involved more than the seven professions listed above, they were included only if they focused primarily on nurses, physicians, or allied health professionals. For studies with multiple professions, when possible, we included only the results pertaining to the seven professions above. Studies were excluded if they focused solely on pre-licensure students. We had no historical date limits.

DT independently screened titles and abstracts. Articles that met inclusion criteria were then reviewed a second time using full text. If questions arose related to article eligibility, a second author (DS) reviewed the article. The second author (DS), who is familiar with the complexity literature, reviewed the final list of included studies. The list of articles was sent to a third party expert in the field of complexity for review. All studies were imported into and managed with bibliographic software (Zotero $\left.{ }^{\text {stu }}\right)$.

\section{Charting the data}

Consistent with Arksey and O'Malley [25], we extracted data related to answering our research questions. Data was entered into a Microsoft Excel spreadsheet and individual tables constructed for analysis. Data included authorship, publication year, country of research, research design, professions involved, setting of research (e.g., long term care, acute care), interprofessional focus, purpose/ objective of research, attributes of complexity theory used, phenomena of interest, how complexity theory was used, and definition/description of complexity theory provided. In keeping with a scoping review approach, we did not assess the methodological quality of included studies.

\section{Collating results}

According to Arksey and O'Malley [25], a framework should be used to collate results. We created a framework guided by our five research questions. First, we created a data table for study characteristics, including first author, year published, country, and study design. Second, we created a data table outlining the professions involved, the area of research, the setting of research, and whether the research focused on interprofessional collaboration or education. From these tables we compared characteristics, setting, and profession across all studies to answer our first two research questions. Third, we categorized studies based on their research purpose using the verb presented by the researcher(s) in their purpose statement (e.g., describe, explain, explore). While verbs may overlap when referring to research purposes (e.g., describe and explore), we categorized based on how the authors described their purpose regardless of potential overlap to minimize subjective interpretation of purpose. We then determined each researcher's phenomena of interest. Specifically, we reviewed all research purposes and identified common phenomena of interest. This provided us with a means to categorize studies by research purpose and then compare how the phenomena of interest differed within and between each category thus answering our third research question. Fourth, we reviewed each study and identified how researchers used complexity theory in their study (e.g., conceptual framework, data analysis, interpret findings). Collectively, this approach allowed us to answer our fourth research question. Finally, we created a data table containing the description of complexity theory from each study. From this, we determined the attributes of complexity theory used by each group of authors. To organize the attributes, we followed an approach used by Wallis [13] in his review of complexity in the organizational theory literature. Specifically, we extracted descriptions of the attributes (i.e., conceptual components) of complexity from the definitions and descriptions provided by the authors of the studies in our review and grouped attributes together when authors were describing the same thing. For example, we combined relationships and connections as one attribute: relationships.. We then looked for common themes between descriptions.

\section{Results}

Figure 1 provides an overview of the search and retrieval results. 3478 citations were found by our search strategy. After reviewing titles and abstracts, 792 articles remained. Full text review resulted in 104 articles and after removal of duplicates $(n=55)$ and citations searching $(n=5), 44$ articles were included in our review. Common reasons for study exclusion included: (a) the article was a commentary or debate on the use of complexity theory, (b) the authors used complexity theory to describe an aspect of a disease (e.g., the neural pathway 


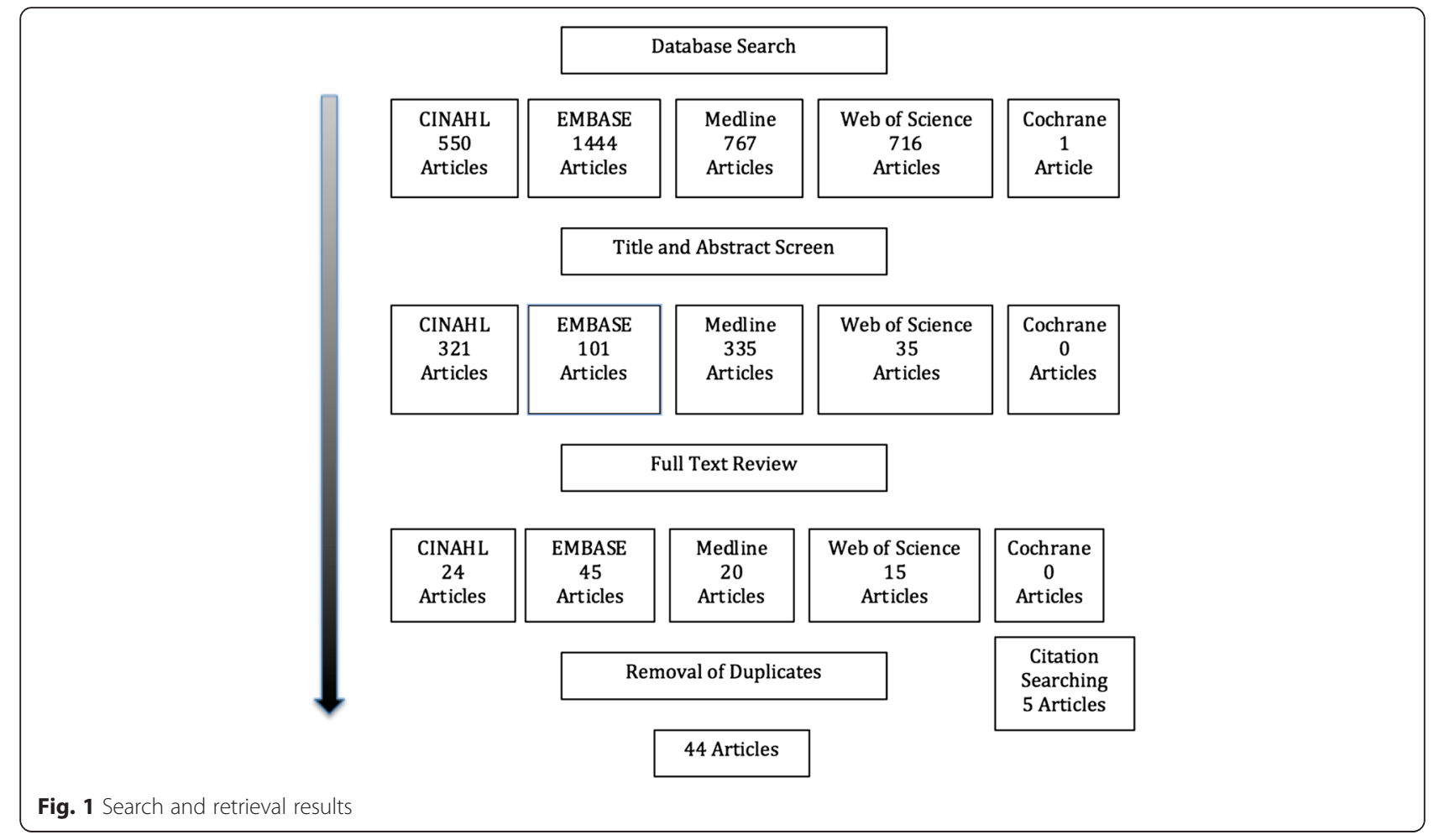

changes of Parkinson's Disease), (c) the study included participants not in our inclusion criteria (e.g., prelicensure learners, administrators) or (d) the research focus not related to health services research (e.g., acoustic properties in rabbits within the context of hearing and speech research).

\section{Characteristics of studies using complexity theory}

The general characteristics of studies incorporating complexity theory in health services research are outlined in Table 2. Most studies were qualitative [31-56], followed by quantitative [57-70], and, finally, to a lesser extent, mixed methods [71-73]. Case studies were the most common qualitative $[31,32,34-37,40-42,44,45,47$, 50, 51, 55, 63, 74] and mixed method [71-73] design. Action research [48], ethnography [39], grounded theory $[46,53]$, and phenomenological designs [43, 49] were used less frequently. Two authors did not identify a specific qualitative design $[54,56]$. There was a mix of designs across the quantitative studies including, in order of frequency, cross-sectional [57, 58, 60, 66, 69], randomized controlled trials [61, 62, 70], retrospective [64, 67], prospective cohort [63], systematic review [65], and unclear [68].

The majority of health services research conducted using complexity theory was based in the United States $[31,32,34,36,37,42,43,46,50-63,65,66,68-70,73$, 74], followed by the United Kingdom [40, 41, 49, 64, 71], Canada [44, 56], Norway [33, 39], Brazil [38], Congo
[45], Finland [67], Italy [47], Malta [35], South Africa [48], and Sweden [72].

Settings and professions studied using complexity theory All of the seven professions listed in our inclusion criteria were represented in our review. Authors in $70 \%$ of the studies included more than the seven professions that comprised our inclusion criteria, with management being the most common group in addition to our inclusion criteria. Studies including nursing were most frequent $(82 \%)$ followed by studies including physicians (52\%).

The settings studied using complexity theory consisted of long term care facilities [32, 34, 36, 37, 39, 42, 51, 54, $57,59-61,69,70]$, primary care $[31,33,40,41,44,46$, $47,50,55,62,68,71]$, hospital $[35,38,43,45,52,53,58$, $63,64,66,67,73,74]$, community health centres $[48,62$, $74]$, and other (e.g., not applicable, health care systems, health trusts) $[49,56,65,72]$. Despite most of the research being conducted with multiple professions and in settings that depend upon interprofessional collaboration, only $23 \%$ of studies used complexity theory to explicitly explore interprofessional collaboration.

\section{Research purpose and phenomena of interest}

Authors used a variety of research purposes to study an assortment of phenomena using complexity theory. See Table 3 for research purposes and phenomena grouping for all studies. The most common research purpose was 
Table 2 Study characteristics, application, and attributes of complexity theory in health services research

\begin{tabular}{|c|c|c|c|c|c|c|c|}
\hline First author & Year & Country & Setting & Professions involved & Research design & Use of complexity theory & Attributes of complexity theory used \\
\hline Aita [31] & 2005 & USA & Primary Care & Physicians & $\begin{array}{l}\text { Qualitative-Secondary Analysis } \\
\text { of a Comparative Case Study }\end{array}$ & Data analysis & Attractors \\
\hline Anderson [57] & 1999 & USA & Long Term Care & Nurses & Quantitative-Cross Sectional & $\begin{array}{l}\text { Conceptual framework } \\
\text { and interpretation of } \\
\text { findings }\end{array}$ & $\begin{array}{l}\text { Communication, Connections, } \\
\text { Diversity }\end{array}$ \\
\hline Anderson [58] & 2003 & USA & Hospital & $\begin{array}{l}\text { Nurses, Pharmacists, } \\
\text { and Physicians }\end{array}$ & Quantitative-Cross Sectional & Conceptual framework & Connections, Diversity, Feedback \\
\hline Anderson [59] & 2003 & USA & Long Term Care & Nurses & Quantitative-Cross Sectional & Conceptual framework & $\begin{array}{l}\text { Communication, Connections, } \\
\text { Diversity, Self-Organization }\end{array}$ \\
\hline Anderson [60] & 2004 & USA & Long Term Care & Nurses & Quantitative-Cross Sectional & Conceptual framwork & $\begin{array}{l}\text { Connections, Communication, } \\
\text { Self Organization }\end{array}$ \\
\hline Anderson [32] & 2014 & USA & Long Term Care & Nurses & $\begin{array}{l}\text { Qualitative-Comparative } \\
\text { Case Study }\end{array}$ & $\begin{array}{l}\text { Conceptual framework } \\
\text { and interpret findings }\end{array}$ & $\begin{array}{l}\text { Communication, Connections, } \\
\text { Diversity, Emergence, Non-Linearity, } \\
\text { Self-Organization }\end{array}$ \\
\hline Brandstorp [33] & 2015 & Norway & Primary Care & Nurses and Physicians & Qualitative-Action Research & Data analysis & $\begin{array}{l}\text { Attractors, Adaptation, Emergence, } \\
\text { Feedback, Self-Organization }\end{array}$ \\
\hline Brannon [34] & 2009 & USA & Long Term Care & Nurses & Qualitative-Case Study & Data analysis & $\begin{array}{l}\text { Agents, Connections, Diversity, } \\
\text { Emergence, Feedback, Self- } \\
\text { Organization }\end{array}$ \\
\hline Buttigieg [35] & 2013 & Malta & Rehab Hospital & $\begin{array}{l}\text { Physiotherapists, } \\
\text { Occupational Therapists, } \\
\text { Nurses, Pharmacists, }\end{array}$ & Qualitative_Case Study & Conceptual framework & Unclear \\
\hline Colón-Emeric [61] & 2013 & USA & Long Term Care & Nurses & $\begin{array}{l}\text { Quantitative-Cluster } \\
\text { Randomized Control Trial }\end{array}$ & Conceptual framework & $\begin{array}{l}\text { Communication, Connections, } \\
\text { Diversity, Self-Organization }\end{array}$ \\
\hline Colón-Emeric [36] & 2006 & USA & Long Term Care & $\begin{array}{l}\text { Dieticians, Nurses, } \\
\text { Physiotherapists, } \\
\text { Occupational Therapists }\end{array}$ & Qualitative-Case Study & Data analysis & $\begin{array}{l}\text { Adaptation, Communication, } \\
\text { Diversity, Self-Organization }\end{array}$ \\
\hline Cólon-Emeric [37] & 2006 & USA & Long Term Care & Nurses and Physicians & $\begin{array}{l}\text { Qualitative-Comparative } \\
\text { Case Study }\end{array}$ & Conceptual framework & $\begin{array}{l}\text { Connections, Communication, } \\
\text { Diversity, Learning }\end{array}$ \\
\hline Cucolo [38] & 2015 & Brazil & Hospital & Nurses & Qualitative-Content Analysis & Data analysis & Unclear \\
\hline Dickinson [62] & 2014 & USA & $\begin{array}{l}\text { Community Health } \\
\text { Centres and Primary } \\
\text { Care }\end{array}$ & Physicians & $\begin{array}{l}\text { Quantitative_Cluster } \\
\text { Randomized Control Trial }\end{array}$ & Conceptual framework & $\begin{array}{l}\text { Adaptation, Connections, } \\
\text { Diversity, Learning, Reflection }\end{array}$ \\
\hline Eika [39] & 2015 & Norway & Long Term Care & Nurses & Qualitative_Ethnography & Conceptual framework & $\begin{array}{l}\text { Emergence, Learning, Self- } \\
\text { Organization }\end{array}$ \\
\hline Ellis [40] & 2010 & United Kingdom & Primary Care & Nurse and Physicians & $\begin{array}{l}\text { Qualitative-Comparative } \\
\text { Case Study }\end{array}$ & Interpret findings & $\begin{array}{l}\text { Adaptation, Agents, Co-Evolution, } \\
\text { Self-Organization }\end{array}$ \\
\hline Ellis [71] & 2011 & United Kingdom & Primary Care & Nursing and Physicians & Mixed Methods_Case Study & Interpret findings & $\begin{array}{l}\text { Agents, Co-Evolution, Emergence, } \\
\text { Self-Organization }\end{array}$ \\
\hline Ellis [41] & 2011 & United Kingdom & Primary Care & Nursing and Physicians & Qualitative-Case Study & Interpret findings & $\begin{array}{l}\text { Adaptation, Agents, Co-Evolution, } \\
\text { Self-Organization }\end{array}$ \\
\hline
\end{tabular}


Table 2 Study characteristics, application, and attributes of complexity theory in health services research (Continued)

\begin{tabular}{|c|c|c|c|c|c|c|c|}
\hline Erdek [63] & 2004 & USA & Hospital & Nurses and Physicians & $\begin{array}{l}\text { Quantitative_Prospective } \\
\text { Cohort Study }\end{array}$ & Interpret findings & Unpredictability \\
\hline Essen [72] & 2013 & Sweden & $\begin{array}{l}\text { Rheumatology } \\
\text { Registry }\end{array}$ & Physicians and Nurses & Mixed Methods_Case Study & $\begin{array}{l}\text { Data analysis and } \\
\text { interpret findings }\end{array}$ & $\begin{array}{l}\text { Equilibrium, Emergence, Feedback, } \\
\text { Self-Organization }\end{array}$ \\
\hline Forbes-Thompson [42] & 2007 & USA & Long Term Care & Nurses & Qualitative-Case Study & Data analysis & $\begin{array}{l}\text { Communication, Connections, } \\
\text { Diversity }\end{array}$ \\
\hline Ford [73] & 2009 & USA & Hospital & Nurses & Mixed Methods_Case Study & Interpret findings & Diversity, Emergence, Relationships \\
\hline Glenn [43] & 2014 & USA & Hospital & Nurses & $\begin{array}{l}\text { Qualitative_Hermeneutic } \\
\text { Phenomenology }\end{array}$ & $\begin{array}{l}\text { Conceptual framework } \\
\text { and interpret findings }\end{array}$ & $\begin{array}{l}\text { Agents, Decentralized Control, } \\
\text { Emergence, Feedback, Non- } \\
\text { Linearity, Self-Organization }\end{array}$ \\
\hline Haigh [64] & 2008 & United Kingdom & Hospital & Nurses & $\begin{array}{l}\text { Quantitative_-Retrospective } \\
\text { Statistical Modeling }\end{array}$ & Equation to predict changes & $\begin{array}{l}\text { Attractors, Equilibrium, Non- } \\
\text { Linearity }\end{array}$ \\
\hline Hilts [44] & 2013 & Canada & Primary Care & Physicians & Qualitative_Case Study & Data analysis & $\begin{array}{l}\text { Communication, Emergence, } \\
\text { Reflection }\end{array}$ \\
\hline Karemere [45] & 2015 & Congo & Hospitals & Physicians & Qualitative-Case Study & Data analysis & $\begin{array}{l}\text { Agents, Path Depenedency, } \\
\text { Transition Phase }\end{array}$ \\
\hline Lanham [46] & 2009 & USA & Primary Care & Physicians & $\begin{array}{l}\text { Qualitative-Secondary } \\
\text { Analysis Grounded Theory }\end{array}$ & Data analysis & $\begin{array}{l}\text { Agents, Connections, Diversity, } \\
\text { Emergence, Learning }\end{array}$ \\
\hline Lanham [74] & 2013 & USA & $\begin{array}{l}\text { Hospitals and } \\
\text { Community }\end{array}$ & Nurses & Qualitative_Case Study & Interpret findings & $\begin{array}{l}\text { Connections, Learning, Self- } \\
\text { Organization }\end{array}$ \\
\hline Leykum [65] & 2007 & USA & Not Applicable & $\begin{array}{l}\text { Studies that would } \\
\text { include at minimum } \\
\text { nurses and physicians }\end{array}$ & $\begin{array}{l}\text { Quantitative-Systematic } \\
\text { Review }\end{array}$ & Classification & $\begin{array}{l}\text { Co-Evolution, Connections, Learning, } \\
\text { Self-Organization }\end{array}$ \\
\hline Longo [47] & 2007 & Italy & Primary Care & Physicians & Qualitative-Case Study & $\begin{array}{l}\text { Conceptual framework } \\
\text { and interpret findings }\end{array}$ & Learning, Relationships \\
\hline Mash [48] & 2008 & South Africa & Community Health & Nurses and Physicians & Qualitative-Action Research & Interpret findings & $\begin{array}{l}\text { Emergence, Self-Organization, } \\
\text { Relationships }\end{array}$ \\
\hline Matthews [49] & 2007 & United Kingdom & Health Trusts & $\begin{array}{l}\text { Nurses, Physicians, } \\
\text { Pharmacists }\end{array}$ & Qualitative_Phenomenology & $\begin{array}{l}\text { Conceptual framework } \\
\text { and intertpret findings }\end{array}$ & $\begin{array}{l}\text { Agents, Diversity, Emergence, } \\
\text { Feedback, Non-Linearity, Self- } \\
\text { Organization }\end{array}$ \\
\hline Miller [50] & 2001 & USA & Primary Care & Nurses and Physicians & $\begin{array}{l}\text { Qualitative-Comparative } \\
\text { Case Study }\end{array}$ & Data analysis & $\begin{array}{l}\text { Co-Evolution, Emergence, Self- } \\
\text { Organization }\end{array}$ \\
\hline Oyeleye [66] & 2013 & USA & Hospital & Nurses & Quantitative-Cross-Sectional & Conceptual framework & Agents, Non-Linearity, Relationships \\
\hline Pitkäaho [67] & 2015 & Finland & Hospital & Nurses & Quantitative-Retrospective & Conceptual framework & $\begin{array}{l}\text { Feedback, Non-Linearity, } \\
\text { Relationships }\end{array}$ \\
\hline Piven [51] & 2006 & USA & Long Term Care & Nurses & Qualitative_Case Study & Data analaysis & $\begin{array}{l}\text { Communication, Connections, } \\
\text { Diversity }\end{array}$ \\
\hline Provost [52] & 2015 & USA & Hospitals & $\begin{array}{l}\text { Nurses, Pharmacists, } \\
\text { Physicians }\end{array}$ & Qualitative_Field Study & Conceptual framework & $\begin{array}{l}\text { Communication, Learning, } \\
\text { Relationships }\end{array}$ \\
\hline Rangachari [53] & 2008 & USA & Hospital & Physicians & Qualitative-Grounded Theory & Conceptual framework & Attractors, Diversity, Emergence \\
\hline
\end{tabular}


Table 2 Study characteristics, application, and attributes of complexity theory in health services research (Continued)

\begin{tabular}{|c|c|c|c|c|c|c|c|}
\hline Rantz [54] & 2013 & USA & Long Term Care & Nurses & Qualitative_-Unclear & $\begin{array}{l}\text { Conceptual framework } \\
\text { and data analysis }\end{array}$ & $\begin{array}{l}\text { Connnections, Communication, } \\
\text { Emergence, Self-Organization }\end{array}$ \\
\hline Rantz [70] & 2012 & USA & Long Term Care & Nurses & $\begin{array}{l}\text { Quantitative-Randomized } \\
\text { Controlled Trial }\end{array}$ & Conceptual framwork & $\begin{array}{l}\text { Communication, Connections, } \\
\text { Diversity }\end{array}$ \\
\hline Ruhe [55] & 2005 & USA & Primary Care & Physicians & Qualitative-Case Study & Data analysis & $\begin{array}{l}\text { Communication, Connections, } \\
\text { Diversity, Emergence, Equilibrium, } \\
\text { Feedback }\end{array}$ \\
\hline Singh [68] & 2004 & USA & Primary Care & Nurses and Physicians & Quantitative-Unclear & Conceptual framework & $\begin{array}{l}\text { Adaptation, Central Attractors, } \\
\text { Communication, Diversity }\end{array}$ \\
\hline Sterns [69] & 2010 & USA & Long Term Care & Nurses & Quantitative-Cross Sectional & Classification & Agents, Unpredictability \\
\hline Tsasis [56] & 2012 & CAN & Health Care System & Nurses and Physicians & Qualitative_-Unclear & Data analysis & $\begin{array}{l}\text { Agents, Co-Evolution, Diversity, } \\
\text { Emergence, Non-Linearity, } \\
\text { Self-Organization }\end{array}$ \\
\hline
\end{tabular}

Note: Only the professions outlined in our elegibility criteria are reported in Table 2 
Table 3 Research purpose and phenomena of interest

\begin{tabular}{|c|c|}
\hline Research purpose & Phenomena of interest \\
\hline Exploratory & $\begin{array}{l}\text { Change [41, 44], Leadership [33], Management } \\
{[48,58,60] \text {, Interactions/Relationships }[39,49,52 \text {, }} \\
53,57,59] \text {, Working environment }[43,44,66]\end{array}$ \\
\hline Describe & $\begin{array}{l}\text { Interactions/Relationships [32, 36, 37, 51, 54], } \\
\text { Management [32, 36, 40], Working environment [42] }\end{array}$ \\
\hline Examine & Change $[65,69,74]$, Interactions/Relationships [31] \\
\hline $\begin{array}{l}\text { Combined other } \\
\text { purposes }\end{array}$ & $\begin{array}{l}\text { Change }[34,47,55,56,62,68,70-72] \text {, Management } \\
{[35,41,64,73] \text {, Interactions/Relationships }[46,61,67] \text {, }} \\
\text { Working Environment }[38,45,50,56]\end{array}$ \\
\hline
\end{tabular}

exploratory (30\%). Of these, $69 \%$ of studies listed a second purpose (to test, to describe, to develop, to examine, to identify). We further grouped exploratory studies into categories based on their phenomena of study (Table 3). These included interactions/relationships (e.g., participation in decision making [57]), management (e.g., management practices on staff turnover [60]), working environment (e.g., staff perspectives on caring practices [43]), and leadership (e.g., training teams [33]). Two studies had two phenomena of interest based on our coding scheme $[44,58]$. Authors of one study [58] explicitly focused on both management and interactions/ relationships and the other study [44] explicitly focused on working conditions and change. All of the exploratory studies involving interactions/relationships focused on health professionals.

Research purposes aimed at describing phenomena were the second most common (16\%). Of these, two studies [36, 51] listed a second purpose of exploring. Similar to the exploratory studies, we grouped studies based on the phenomena of interest. Similar to the exploratory studies, the majority of descriptive studies aimed to describe an aspect of interaction/relationships (e.g., describe staff behaviour in group processes [54]) between health professionals either as a primary aim or as a combined aim with management (e.g., describe connection patterns among staff [36]). One study described aspects solely related to management (e.g., clinical governance, management practices [40]) and one study described aspects solely related to work environment (e.g., describe working conditions in nursing homes [42]).

Research purposes aimed at examining phenomena were the third most common (9\%). Due to the low number of studies, we narratively report the results. The first group of authors [31] examined interactions/ relationships. Specifically, they examined features of practice related to patient centeredness using a secondary analysis of qualitative data. They concluded that attributes of complexity theory assisted them in examining how patient centeredness occurs within patient and physician interactions. The second group of authors [65] examined change. They conducted a systematic review of interventions aimed at improving Type II diabetes. The authors assigned a value to each intervention based on the degree of complexity that the intervention exhibited. The authors used the degree of complexity to examine whether interventions based on complexity attributes were more effective than interventions that were not based on complexity. They concluded that interventions with a greater number of complexity attributes were more effective for changing diabetic outcomes. The third group of authors [69] also examined change. These authors examined the degree of culture change practice adoption. They ranked culture change practices based on their degree of complexity and examined the degree of adoption. The authors concluded that less complex practices may be easier to implement and that implementation of less complex practices may improve implementation of more complex changes. Finally, Lanham and colleagues [74] used several attributes of complexity theory to re-examine two studies that evaluated the spread of effective interventions. They concluded that self-organization, sense making, and interconnections could be used to facilitate the spread of effective practices.

The heterogeneity of research purposes included in the remaining studies ( $45 \%$ ) prevented meaningful comparison. The research purposes that authors reported include advance and understand, analyze, compare, demonstrate, determine, document, estimate impact, evaluate, identify, implement, improve, produce, suggest, test hypothesis, and understand. We categorized these studies based on phenomena of interest. Change was the most common focus of studies within this category, followed by work environment, management, and, finally, interactions/relationships.

In summary, based on our analysis of research purpose and phenomena of interest, studies aimed at exploring and studies aimed at describing represent the most common research purpose of health services research incorporating complexity theory. Within these categories, complexity theory was incorporated primarily to explore or describe interactions/relationships between health care workers. There is a wide range of research purposes in the remaining studies. Within these remaining studies, the most common phenomenon of interest was change.

\section{Use of complexity theory in health services research}

Researchers have used complexity theory in their research in a variety of ways (Table 2). The most common was as a conceptual framework applied to research approach and design (45\%). ${ }^{2}$ Examples include using complexity theory to conceptualize variables that were subsequently operationalized to determine if attributes of complexity account for rates in staff turnover [60], 
using complexity theory to conceptualize the work environment [43], and using complexity theory to conceptualize primary care organizations [40]. There was variation on how explicit authors were regarding how they used complexity theory as a conceptual framework. Some authors described in detail the attributes they used and how they used them, whereas others stated that their research incorporated a complexity framework without describing which attributes or how complexity was used (e.g., [45]).

The second most common use of complexity theory was as a framework for data analysis (32\%). In this group, all studies were qualitative designs and the majority $(57 \%)$ were case studies with authors using attributes of complexity to in data analysis. Examples of how complexity theory were used to in data analysis include comparing attributes of complexity (e.g., self organization, emergence) across case studies [50], using complexity to "understand what we were seeing" [31], and using complexity to code observations [34]. Again, similar to those that used complexity as a conceptual framework, authors who used complexity as a data analysis framework varied in detail regarding what they used and how they used it.

Finally, the third most common use of complexity theory was as a framework for interpreting findings (29\%). Examples include using complexity to illustrate leadership principles [73], explain clinical governance [40], and hypothesize why an intervention worked to improve pain control [63].

The remaining three studies used complexity to predict change [64] or classify either interventions [65] or culture change practices [69]. Several authors reported dual applications of complexity (e.g., [47]) and we included both applications in our results (Table 2).

\section{Descriptions of complexity theory}

Authors have incorporated a wide range of attributes from complexity theory to study phenomena related to health services research. To facilitate analysis, we grouped certain attributes into categories when authors appeared to refer to the same (or similar) concept of complexity. Table 4 lists the referent attributes we combined and the term we used to refer to the parent attribute. Wallis [18] used a similar approach in his review of complexity theory in organizational science. As complexity theory has no agreed upon definition and a myriad of concepts that comprise the theories subsumed within complexity theory, it was necessary to combine certain attributes to facilitate analysis. Furthermore, it is beyond the scope of this review to offer a definition of each attribute. However, readers interested in definitions/descriptions of attributes of complexity may be interested in referring to The Handbook of Systems and Complexity in Health [75].
Table 4 Parent and referrent attributes

\begin{tabular}{|c|c|}
\hline Parent attribute & Referent attributes \\
\hline Connections & Connections, Relationships, Interconnections \\
\hline Communication & $\begin{array}{l}\text { Communication, Conversation, Information Flow, } \\
\text { Information Exchange, Interactions }\end{array}$ \\
\hline Learning & Learning, Sense Making, Learning Culture \\
\hline Adaptation & Adaptation, System Adaptation, Innovation \\
\hline Diversity & $\begin{array}{l}\text { Diversity, Cognitive Diversity, Diversity of Information, } \\
\text { Diversity of Perspective, Diversity of Views }\end{array}$ \\
\hline Equilibrium & Equilibrium, Disequilibrium \\
\hline Agents & Agents, Agents in a System, Input from Agents \\
\hline Unpredictability & Unpredictability, Uncertainty, Levels of Certainty \\
\hline
\end{tabular}

Overall, researchers incorporated a total of 18 attributes when referring to complexity theory (Table 2). All of the studies except for two [31, 63] incorporated a combination of attributes. Aita and colleagues [31] incorporated the concept of attractors to interpret secondary data and explore what is involved in patient-centered care within primary care settings. Erdeck and Pronovost [63] introduced an intervention aimed at improving pain management that incorporated the concept of unpredictability (i.e., varying levels of certainty). Notably, in two studies, it was unclear what attributes of complexity the authors used [35, 38].

A combination of three or four attributes of complexity theory was most common. The most attributes incorporated by a group of authors was six. This was done by six groups of authors $[32,34,43,49,55,56]$. Within this group, emergence was included in all studies, followed by self-organization, feedback, agents within a system, non-linearity, and diversity. The remaining attributes appeared once or twice in various combinations.

For all studies included in this review, the most common attributes of complexity theory were relationships $(n=21)$, self organization $(n=19)$, diversity $(n=19)$, emergence $(n=16)$, communication $(n=14)$, feedback $(n=8)$, agents within a system $(n=8)$, and non-linearity $(n=7)$. Descriptions and/or definitions of the attributes varied immensely across studies and it was difficult to know for certain if authors were referring to the same concept when using the same terminology.

Although descriptions of complexity theory varied immensely across studies, it appears authors are describing complexity theory using aspects of the theory that capture how diverse relationships and communication between agents of a system can influence unpredictable changes within the system. It comes as no surprise that descriptions often incorporate relationships, diversity, and communication. Likewise, descriptions also incorporate complexity attributes related to unpredictable changes with self-organization, emergence, and nonlinearity being common in descriptions. The importance 
of capturing relationships and how those relationships contribute to changes in the overall system are apparent in the following examples of direct quotes of author descriptions:

Change emerges through self-organization, defined as the mutual adjustment of behavior arising from interactions among staff as they meet immediate care demands [51].

Complexity science suggests that organizations, such as hospitals, are complex adaptive systems. As such, a hospital is defined as a set of connected or interdependent parts or agents-including caregivers and patients - bound by a common purpose and acting on their knowledge [58].

Complexity science, as related to healthcare, is the science of moving in a nonlinear and interactive manner where unpredictable outcomes are often realized; organizations are described as ever-changing collections of individuals and conditions in the organization; and patterns of interaction among individuals and connections are made in day-to-day practices among and between individuals [66].

Despite not knowing if authors are referring to the same thing when they use similar attributes, these three quotes of authors' descriptions of complexity in health services research typify a common thread in the studies included in our review. In some cases, descriptions of complexity theory in health services research incorporate the theory's ability to view communication and relationships between diverse agents in a system as supporting factors to overall changes of the system.

\section{Discussion}

This is the first scoping review to explore how complexity theory has been incorporated into health science research. Studies incorporating complexity theory appear to be increasing in frequency. Health services researchers are primarily using complexity theory with qualitative case studies conducted in the US focused on nursing and medicine in long-term care and primary care. Quantitative and mixed methods studies using complexity theory exist, and other settings are being studied, but both to a lesser extent. Research is primarily exploratory or descriptive in nature and aimed at understanding phenomena related to interactions/relationships and management. Descriptions of complexity theory varied with 18 attributes of complexity theory across all studies in this review. The most common attributes were relationships, self-organization, and diversity. Descriptions appear to focus on aspects of complexity theory related to how diverse relationships and communication between individuals in a system may influence change.
There is notable consistency between our findings and existing reviews. Similar to Sturmberg et al.'s [20] review of complexity in family medicine general practice, we found health services researchers to be expanding how they incorporate complexity theory in research. However, this expansion has largely remained at exploratory and descriptive level of research. In a review of complexity in computer science, mathematics, and management research, Pollack et al. [19] used referencing patterns and concluded that the application of complexity theory to organizational science research using mathematical modeling techniques is uncommon. Sturmberg et al. [20] reported similar findings in family medicine general practice. Despite 14 studies in our review being quantitative, there was minimal mathematical modeling. Although some studies in our review used modeling (see for example [57, 64], mathematical modeling using complexity theory does not appear common in health services research and the use of complexity theory remains at a descriptive or exploratory level. This is not surprising since complexity theory is primarily used as an explanatory theory as opposed to predictive one [76].

Pollack et al. [19] and Sturmberg et al. [20] recommend authors move beyond metaphorical application of complexity as an observation tool. Both suggest a mathematical basis of inquiry is possible to progress complexity's application within social sciences research. They argue a shift would enable researches to use complexity theory as a basis for quantitative modeling. Notably, neither group contends quantitative-modeling should occur without using complexity's metaphors as building blocks for conceptual frameworks; these methodological approaches are complementary and complexity is useful for each. Although we agree with Pollack et al. [19] and Sturmberg et al. [20], we offer cautionary advice. Our findings demonstrate variation in how authors are incorporating complexity theory in health services research with a broad range of attributes being used. Thus, we align ourselves with Greenhalgh and colleagues [21] and suggest more adaptation and refinement is needed to determine how a complexity perspective can be used to answer health services research questions. That is not to say mathematical modeling is not useful. However, forgoing foundational work and shifting methodological approaches will not progress complexity's usefulness to health services research and may only lead to more conceptual confusion. As our review suggests, there is too much variation to be certain authors are talking about, even at a metaphorical level, the same concept.

In a review of complexity in organizational science, Wallis [13] identified 20 definitions of complex adaptive systems containing 26 different conceptual components. We found authors within our review used 18 different attributes of complexity theory. Although we used 
different labels than Wallis, overlap exists between common attributes used in organizational science and those used in health services research. Self-organization, agents, emergence, non-linearity, and interacting/relationships were among the most common in both reviews. Likewise, descriptions that focused on how diverse relationships and communication contributed to changes within a system are predominant. Using the most common collective attributes as an indicator for what researchers consider the most applicable components of complexity theory within a social sciences context provides a foundation to begin to develop a better understanding of each concept and how it can be used to comprise a complexity theory perspective in health services research. Such foundational work is imperative. Many authors (e.g., [22, 75, 77] agree that complexity theory offers a useful perspective to answer questions of a social nature. Likewise, many authors (e.g., [53-55]) agree that descriptions of complexity theory are varied and influenced by discipline and phenomena of interest. Given complexity theory's application in health services research is relatively new compared to other fields, health services researchers have a unique opportunity to develop the foundational conceptual perspectives that complexity theory offers health services research.

Davis and colleagues [15] suggest complexity theory is not a theory but more a perspective or way of thinking about certain phenomena. They argue that the transdisciplinary nature of a complexity perspective prevents an "off the shelf" definition and application. Although the transdisciplinary nature of complexity cannot be argued, the results of our scoping review and other reviews of complexity (i.e., $[13,20]$ ) provide a glimpse of caution that should be considered when working with complexity. Indefinable theoretical perspectives can lead to studies with unclear or missing descriptions, implicit assumptions, and absent definitions. As a result, findings from such studies are difficult to generalize with confidence. Of course, all theories, especially transdisciplinary ones, require users to assume relationships that are, perhaps, untested. Consider Rogers' innovation diffusion theory (a transdisciplinary theory) is the most influential theoretical perspective in the knowledge translation [78]. However, its use in knowledge translation, specifically health, requires an untested assumption that knowledge application in health is akin to classical diffusion theory [79]. Such an assumption has not limited the theory's usefulness; however, it is worth considering in the realm of complexity how many assumptions and varied definitions are tolerable.

A lack of description of how complexity is used in original research creates challenges for drawing conclusions across health services research using review methodologies (e.g., scoping, systematic, narrative). For example, we excluded several studies where authors did not explicitly state they used complexity theory in their original manuscripts. This may have resulted in research that incorporated complexity from being excluded from our review. For example, Crabtree and colleagues have conducted a longstanding program of research using complexity theory that they outlined in a 2011 publication [80]. Such work represents a substantial contribution. However, when assessing some of Crabtree and colleagues' original studies which form the basis of the 2011 publication (i.e. [81-85] using our inclusion/exclusion criteria, we could not include the studies because the authors did not explicitly state they used complexity theory in the original manuscripts, they did not explicitly discuss complexity theory in their original manuscripts, and it was a subsequent publication [80] that identified the studies as using complexity theory.. Notably, these studies were not captured by our search strategy because they were not indexed using medical subject headings $(\mathrm{MeSH})$ related to complexity nor did they have complexity as key words or titles. Consequently, they were captured by citation searching key articles located by our database searches. While such research has the potential to advance our understanding how to use complexity to answer important health services research questions, without clear and explicit descriptions of how complexity theory was used a priori in designing a study, it is difficult to know how to use complexity theory to design future studies. Notwithstanding, papers by original authors offering a retrospective look back on their program of research from a complexity theory lens are helpful (i.e., $[80,86]$ but such works are difficult to integrate into reviews by other authors (e.g., this scoping review).

From this review, we stop short of recommending that complexity theory is more appropriate than other theories for incorporating into health services research. Complexity is one of many theories researchers available to health services researchers. However, the findings of our review suggest that for researchers studying factors related to relationships, communication, and diversityspecifically how these factors may contribute to change within a system-other authors have found that complexity offers an appropriate choice.

The appropriateness of complexity theory in studying systems stems from how it allows a researcher to conceptualize a system. Specifically, complexity conceptualizes a system as non-linear and dynamical. Complex systems can be understood by comparison to complicated systems. Briefly, in a complicated system, the parts that comprise the system combine in predictable, knowable ways to comprise the overall system. If one were to conceptualize a health system as complicated, it would be possible to reduce the system and study the individual to gain an understanding of the overall system. If 
one studied enough components, one would know how the system works and therefore how to manipulate the system. Such an approach has fallen short when studying health systems [87]. Instead, complexity theory offers a toolkit (i.e., attributes) for conceptualizing and studying health systems in different manner. Complexity brings to the forefront the unpredictable nature of a complex system. Specifically, according to complexity, systems are still comprised of agents, but those agents interact with each other. The interactions of the agents are decentralized. From these interactions, changes occur within the system that may bring about additional change. One cannot trace the original cause of the change. So, while other theories offer tools for studying systems, many are based on the assumptions that systems behave like a complicated system, are predictable, and can be understood by studying components of a system. The reason we stop short of suggesting complexity is more appropriate than other theories for studying health services research is because health systems are comprised of both complex and complicated systems. In some instances, depending on how the researcher conceptualizes the phenomena of study, theories that assume a complicated system are appropriate. However, instances where complex systems are involved, such as understanding how change may influence organizational culture, complexity theory offers an appropriate perspective.

Complexity theory is similar to other theories useful in health services research-especially theories aimed at exploring relationships in systems. Two such theories are systems theory and social network theory. Authors identify systems theory as being closely related to complexity theory [88-90]. Similar to complexity, systems theory also seeks to understand how relationships between agents of a system influence change. However, according to Phelan [89], systems theory is focused on identifying and optimizing relationship characteristics whereas complexity is focused on understanding what influences interactions so that conditions may be created to support further interactions. In essence, complexity is more exploratory whereas systems theory is more confirmatory [89]. Social network theory offers a perspective of how relationships between individuals can influence the spread of something (e.g., information, disease, innovation) within networks [91, 92]. Using social network theory, researchers can map detailed relationships between entities for the purposes of describing and predicting how network structure may influence an outcome. In essence, the focus in social network theory is the connection of agents within a system. While complexity theory also offers a perspective on connections between agents, the focus of complexity takes a less reductionist view on interactions than social network theory. Complexity theory "counsels that analytical and predictive power can only be gained by standing back-not analyzing a system in more detail" [93]. Clearly systems theory, social network theory, and many other theories are appropriate for health services research. A choice of theory depends on multiple perspectives. As such, we stop short of suggesting complexity theory is more appropriate than other theories align ourselves with Davis and Sumara [90] to suggest complexity does not rise over other theories but instead rises among them.

Variation across studies on how complexity is incorporated is expected. It is a product of intellectual grappling, experimentation, and exploration on how a complexity perspective can be incorporated to answer health services research questions. In a sense, the findings of this scoping review represent evidence that the foundational work that so many authors urge is occurring. Although we are unable to determine what is appropriate use of complexity theory in health services research, the appropriateness of variation in the early stages of complexity applied to health services research is an expected finding of this scoping review.

\section{Limitations}

There are several limitations in our review. First, related to our search strategy, we acknowledge that not all authors will agree our search terms are integral with elements of complexity theory. We felt it necessary to take an approach of broadness during study identification, keeping with Arksey and O'Malley's [25] framework for scoping reviews. Second, this scoping review was conducted as part of a doctoral dissertation. As such, it was conducted primarily independently (with a second reviewer when needed) and, therefore, did not benefit from a team approach to scoping methodology (see for example $[26,94])$. A solitary approach has been used in scoping reviews by other doctoral candidates (e.g., [95]), however; the results would be strengthened by a team of reviewers. Third, we included only studies published in English. The effect of inclusion and exclusion in systematic reviews by language is inconclusive [96], yet there is a possibility of excluding important studies from our scoping review-most likely related to the country of research origin.

\section{Conclusion}

Researchers are incorporating complexity theory in health services research. Researchers using complexity theory in health services research are primarily using the theory for various aspects of qualitative case studies (e.g., conceptual framework for study design, framework for data analysis) involving nursing and medicine in long-term care and primary care. Research is at the 
exploratory or descriptive level and focused on interactions/relationships and management. Authors have employed many attributes of complexity and descriptions often incorporate aspects of complexity theory related to how diverse relationships and communication between individuals in a system can influence change.

The overarching theme from this scoping review is variation. Although variation may be thought of as a drawback, variation may also be a product of applying a novel and malleable theory in a new context. We do not yet know how best to incorporate complexity to study phenomena in health services research and the debate is far reaching. Perhaps there is no one method to apply this theory and its malleability permits broad application? That said, authors are attempting to study important phenomena using complexity theory and are grappling with how to use this theory. Although complexity theory shows promise in health services research and health services delivery, conceptual confusion and inconsistent application hinders the operationalization of this potentially important perspective. Complexity appears particularly applicable for studying relationships and interactions between health professionals and management. However, generalizability from studies that use complexity theory, at present, is difficult due to heterogeneity and variation in reporting. Future research should include clear definitions and descriptions of complexity and how it was used in studies. In summary, more research, debate, and exploration are still needed to continue to understand how complexity theory can be incorporated in health services research.

\section{Endnotes}

${ }^{1}$ The term use in this instance refers to how authors used complexity theory specifically in their study. This differs from the term incorporated, which we use to refer to the broader use of complexity in health services research and to encompass all of our questions.

${ }^{2}$ The percentages in this section do not add up to $100 \%$ because some authors used complexity theory in multiple ways.

\section{Abbreviations \\ CINAHL: cumulative index to nursing and allied health literature; EMBASE: excerpta medica database; MLIS: master of library information science.}

\section{Competing interests}

The authors declare they have no competing interests.

\section{Authors' contributions}

DT was the principal investigator. This study comprised part of the graduate work of DT. DT designed the study, conducted the search, extracted the data, and analyzed the data. DS acted as a second reviewer. DS, EK, LP, XF assisted in review and feedback of the final manuscript. DS served as DT's graduate supervisor. EK, LP, and XF served as DT's supervisory committee. All authors read and approved the final manuscript.

\section{Acknowledgements}

The authors gratefully acknowledge the librarian assistance of Debra Gold for assisting with the search and anonymous reviewers for improving previous drafts.

\section{Author details}

'School of Nursing, Lakehead University, 955 Oliver Road, Thunder Bay, ON P7B 5E1, Canada. 'Faculty of Education, Brock University, 500 Glenridge Avenue, St. Catharines, ON L2S 3A1, Canada. ${ }^{3}$ Teaching and Learning Development, University of Windsor, 401 Sunset Avenue, Windsor, ON N9B 3P4, Canada. ${ }^{4}$ Faculty of Nursing, University of Windsor, 401 Sunset Avenue, Windsor, ON N9B 3P4, Canada. ${ }^{5}$ Faculty of Education, University of Windsor, 401 Sunset, Avenue, Windsor, ON N9B 3P4, Canada.

Received: 5 November 2015 Accepted: 9 March 2016

Published online: 12 March 2016

\section{References}

1. Lapaige V. "Integrated knowledge translation" for globally oriented public health practitioners and scientists: Framing together a sustainable transfrontier knowledge translation vision. J Multidiscip Healthc. 2010:3:33-47.

2. Thistlethwaite J. Interprofessional education: a review of context, learning and the research agenda. Med Educ. 2012;46:58-70.

3. Zwarenstein $\mathrm{M}$, Reeves $\mathrm{S}$. Knowledge translation and interprofessional collaboration: Where the rubber of evidence-based care hits the road of teamwork. J Contin Educ Health Prof. 2006:26:46-54.

4. Davies $P$, Walker AE, Grimshaw JM. A systematic review of the use of theory in the design of guideline dissemination and implementation strategies and interpretation of the results of rigorous evaluations. Implement Sci. 2010;5:14

5. Reeves S, Goldman J, Gilbert J, Tepper J, Silver I, Suter E, et al. A scoping review to improve conceptual clarity of interprofessional interventions. J Interprof Care. 2011;25:167-74.

6. Rycroft-Malone J. Theory and Knowledge Translation: Setting Some Coordinates. Nurs Res. 2007;56(1):S78-85.

7. Suter E, Goldman J, Martimianakis T, Chatalalsingh C, DeMatteo DJ, Reeves S. The use of systems and organizational theories in the interprofessional field: Findings from a scoping review. J Interprof Care. 2012;27:57-64.

8. Graham ID, Tetroe J. KT Theories Research Group: Some theoretical underpinnings of knowledge translation. Acad Emerg Med Off J Soc Acad Emerg Med. 2007;14:936-41.

9. Best A, Holmes B. Systems thinking, knowledge and action: towards better models and methods. Evid Policy J Res Debate Pract. 2010;6:145-59.

10. Best A, Saul J, Willis C. Doing the dance of culture change: complexity, evidence and leadership. Healthc Pap. 2013;13:64-8.

11. Kitson AL. The need for systems change: reflections on knowledge translation and organizational change. J Adv Nurs. 2009;65:217-28.

12. Nunn RJ. Complexity theory applied to itself. Emergence Complex Organ. 2007;9:93

13. Wallis S. Emerging order in CAS theory: mapping some perspectives. Kybernetes. 2008;37:1016-29.

14. Manson SM. Simplifying complexity: a review of complexity theory. Geoforum. 2001;32:405-14

15. Davis B, Sumara D, Luce-Kapler R. Engaging Minds: Changing Teaching in Complex Times. 2nd ed. New York: Routledge; 2007.

16. Cilliers P. Understanding Complex Systems. In: Sturmberg JP, Martin CM, editors. Handbook of Systems and Complexity in Health. New York: Springer; 2013. p. 38.

17. Sturmberg JP, Martin CM. Complexity in health: an introduction. In: Handbook of Systems and Complexity in Health. New York: Springer; 2013. p. 1-17.

18. Wallis SE. The Complexity of Complexity Theory: An Innovative Analysis. Emergence Complex Organ. 2009;11:26-38.

19. Pollack J, Adler D, Sankaran S. Mapping the field of Complexity Theory: A computational approach to understanding changes in the field. Emergence Complex Organ. 2014;16:74-92.

20. Sturmberg JP, Martin CM, Katerndahl DA. Systems and complexity thinking in the general practice literature: an integrative, historical narrative review. Ann Fam Med. 2014;12:66-74. 
21. Greenhalgh T, Plsek P, Wilson T, Fraser S, Holt T. Response to "the appropriation of complexity theory in health care". J Health Serv Res Policy. 2010;15:115-7

22. Paley J. Complex adaptive systems and nursing. Nurs Inq. 2007;14:233-42.

23. Paley J. The appropriation of complexity theory in health care. J Health Serv Res Policy. 2010;15:59-61.

24. Grant MJ, Booth A. A typology of reviews: an analysis of 14 review types and associated methodologies. Health Inf Libr J. 2009:26:91-108.

25. Arksey H, O'Malley L. Scoping studies: towards a methodological framework. Int J Soc Res Methodol. 2005;8:19-32

26. Levac D, Colquhoun $\mathrm{H}, \mathrm{O}$ 'Brien KK. Scoping studies: advancing the methodology. Implement Sci. 2010;5:1-9.

27. Colquhoun HL, Levac D, O'Brien KK, Straus S, Tricco AC, Perrier L, et al. Scoping reviews: time for clarity in definition, methods, and reporting. J Clin Epidemiol. 2014:67:1291-4.

28. Newhouse RP, Pettit JC, Poe S, Rocco L. The slippery slope: differentiating between quality improvement and research. J Nurs Adm. 2006:36:211-9.

29. Morris PE, Dracup K. Quality improvement or research? The ethics of hospital project oversight. Am J Crit Care Off Publ Am Assoc Crit-Care Nurses. 2007;16:424-6.

30. Ethics in research: A Science Lifecycle Approach. [http://www.cihr-irsc.gc.ca/ e/48809.html]. Accessed 24 May 2015.

31. Aita V, Mcllvain H, Backer E, McVea K, Crabtree B. Patient-centered care and communication in primary care practice: what is involved? Patient Educ Couns. 2005:58:296-304.

32. Anderson RA, Toles MP, Corazzini K, McDaniel RR, Colon-Emeric C. Local interaction strategies and capacity for better care in nursing homes: a multiple case study. BMC Health Serv Res. 2014;14:244.

33. Brandstorp H, Kirkengen AL, Sterud B, Haugland B, Halvorsen PA. Leadership practice as interaction in primary care emergency team training. Action Res. 2015;13:84-101.

34. Brannon S, Kemper P, Barry T. North Carolina's direct care workforce development journey: the case of the North Carolina New Organizational Vision Award Partner Team. Health Care Manage Rev. 2009;34:284-93.

35. Buttigieg S, Cassar V, Scully J. From words to action: visibility of management in supporting interdisciplinary team working in an acute rehabilitative geriatric hospital. J Health Organ. 2013;27:618-45.

36. Colón-Emeric C, Lekan-Rutledge D, Utley-Smith Q, Ammarell N, Bailey D, Piven $\mathrm{M}$, et al. Connection, regulation, and care plan innovation: a case study of four nursing homes. Health Care Manage Rev. 2006;31:337-46.

37. Cólon-Emeric C, Ammarell N, Bailey D, Corazzini K, Lekan-Rutledge D, Piven M, et al. Patterns of medical and nursing staff communication in nursing homes: implications and insights from complexity science. Qual Health Res. 2006;16:173-88.

38. Cucolo DF, Perroca MG. Factors involved in the delivery of nursing care. ACTA Paul Enferm. 2015:28:120-4.

39. Eika M, Dale B, Espnes GA, Hvalvik S. Nursing staff interactions during the older residents' transition into long-term care facility in a nursing home in rural Norway: An ethnographic study. BMC Health Serv Res. 2015;15:125.

40. Ellis B. Complexity in practice: understanding primary care as a complex adaptive system. Inform Prim Care. 2010;18:135-40.

41. Ellis B. Complex adaptive systems: a tool for interpreting responses and behaviours. Inform Prim Care. 2011;19:99-104.

42. Forbes-Thompson S, Leiker T, Bleich M. High-performing and lowperforming nursing homes: a view from complexity science. Health Care Manage Rev. 2007:32:341-51.

43. Glenn LA, Stocker-Schnieder J, McCune R, McClelland M, King D. Caring nurse practice in the intrapartum setting: nurses' perspectives on complexity, relationships and safety. J Adv Nurs. 2014;70:2019-30.

44. Hilts L, Howard M, Price D, Risdon C, Agarwal G, Childs A. Helping primary care teams emerge through a quality improvement program. Fam Pract. 2013;30:204-11.

45. Karemere $H$, Ribesse N, Kahindo J-B, Maca J. Referral hospitals in the Democratic Republic of Congo as complex adaptive systems: similar program, different dynamics. Pan Afr Med J. 2015;20:281.

46. Lanham HJ, McDaniel RRJ, Crabtree BF, Miller WL, Stange KC, Tallia AF, Nutting P. How improving practice relationships among clinicians and nonclinicians can improve quality in primary care. Jt Comm J Qual Patient Saf Jt Comm Resour. 2009:35:457-66.

47. Longo F. Implementing managerial innovations in primary care: can we rank change drivers in complex adaptive organizations? Health Care Manage Rev. 2007;32:213-25.
48. Mash B, Mayers P, Conradie H, Orayn A, Kuiper M, Marais J. How to manage organisational change and create practice teams: experiences of a South African primary care health centre. Educ Health Change Learn Pract Medknow Publ Media Pvt Ltd. 2008;21:132.

49. Matthews J, Thomas P. Managing clinical failure: a complex adaptive system perspective. J Health Care Qual Assur. 2007;20:184-94.

50. Miller W, McDaniel R, Crabtree B, Stange K. Practice jazz: understanding variation in family practices using complexity science. J Fam Pract. 2001;50: 872-8.

51. Piven M, Ammarell N, Bailey D, Corazzini K, Colón-Emeric C, Lekan-Rutledge D, Utley-Smith Q, Anderson R. MDS coordinator relationships and nursing home care processes. West J Nurs Res. 2006;28:294-309.

52. Provost SM, Lanham HJ, Leykum LK, McDaniel Jr RR, Pugh J. Health care huddles: Managing complexity to achieve high reliability. Health Care Manage Rev. 2015:40:2-12.

53. Rangachari $P$. The strategic management of organizational knowledge exchange related to hospital quality measurement and reporting. Qual Manag Health Care. 2008;17:252-69.

54. Rantz MJ, Zwygart-Stauffacher M, Flesner M, Hicks L, Mehr D, Russell T, Minner D. The Influence of Teams to Sustain Quality Improvement in Nursing Homes that "Need Improvement". J Am Med Dir Assoc. 2013;14:48-52.

55. Ruhe MC, Weyer SM, Zronek S, Wilkinson A, Wilkinson PS, Stange KC. Facilitating practice change: lessons from the STEP-UP clinical trial. Prev Med. 2005:40:729-34.

56. Tsasis P, Evans JM, Owen S: Reframing the challenges to integrated care: a complex-adaptive systems perspective. Int J Integr Care 2012, 12

57. Anderson R, McDaniel Jr RR. RN participation in organizational decision making and improvements in resident outcomes. Health Care Manage Rev. 1999;24:7-16.

58. Anderson R, Allred C, Sloan F. Effect of hospital conversion on organizational decision making and service coordination. Health Care Manage Rev. 2003:28:141-54.

59. Anderson R, Issel L, McDaniel Jr R. Nursing homes as complex adaptive systems: relationship between management practice and resident outcomes. Nurs Res. 2003;52:12-21.

60. Anderson R, Corazzini K, McDaniel Jr RR. Complexity science and the dynamics of climate and communication: reducing nursing home turnover. Gerontologist. 2004:44:378-88.

61. Colon-Emeric CS, McConnell E, Pinheiro SO, Corazzini K, Porter K, Earp KM, Landerman L, Beales J, Lipscomb J, Hancock K, Anderson RA. CONNECT for Better Fall Prevention in Nursing Homes: Results from a Pilot Intervention Study. J Am Geriatr Soc. 2013;61:2150-9.

62. Dickinson WP, Dickinson LM, Nutting PA, Emsermann CB, Tutt B, Crabtree BF, Fisher L, Harbrecht M, Gottsman A, West DR. Practice Facilitation to Improve Diabetes Care in Primary Care: A Report From the EPIC Randomized Clinical Trial. Ann Fam Med. 2014;12:8-16.

63. Erdek M, Pronovost P. Improving assessment and treatment of pain in the critically ill. J Qual Health Care. 2004;16:59-64.

64. Haigh C. Using simplified chaos theory to manage nursing services. J Nurs Manag. 2008;16:298-304

65. Leykum LK, Pugh J, Lawrence V, Parchman M, Noel PH, Cornell J, McDaniel RR. Organizational interventions employing principles of complexity science have improved outcomes for patients with Type II diabetes. Implement Sci. 2007;2:28

66. Oyeleye O, Hanson P, O'Connor N, Dunn D. Relationship of Workplace Incivility, Stress, and Burnout on Nurses' Turnover Intentions and Psychological Empowerment. J Nurs Adm. 2013;43:536-42.

67. Pitkäaho T, Partanen P, Miettinen M, Vehviläinen-Julkunen K. Non-linear relationships between nurse staffing and patients' length of stay in acute care units: Bayesian dependence modelling. J Adv Nurs. 2015;71:458-73.

68. Singh R, Servoss T, Kalsman M, Fox C, Singh G. Estimating impacts on safety caused by the introduction of electronic medical records in primary care. Inform Prim Care. 2004;12:235-41.

69. Sterns S, Miller S, Allen S. The Complexity of Implementing Culture Change Practices in Nursing Homes. J Am Med Dir Assoc. 2010;11:511-8.

70. Rantz MJ, Zwygart-Stauffacher M, Hicks L, Mehr D, Flesner M, Petroski GF, Madsen RW, Scott-Cawiezell J. Randomized multilevel intervention to improve outcomes of residents in nursing homes in need of improvement. J Am Med Dir Assoc. 2012;13:60-8.

71. Ellis B, Howard J. Clinical governance, education and learning to manage health information. Clin Gov Int J. 2011;16:337-52. 
72. Essen A, Lindblad S. Innovation as emergence in healthcare: unpacking change from within. Soc Sci Med. 2013;93:203-11.

73. Ford R. Complex leadership competency in health care: towards framing a theory of practice. Health Serv Manage Res. 2009;22:101-14.

74. Lanham H, Leykum L, Taylor B, McCannon C, Lindberg C, Lester R. How complexity science can inform scale-up and spread in health care: understanding the role of self-organization in variation across local contexts. Soc Sci Med. 2013;93:194-202.

75. Sturmberg JP, Martin CM. Handbook of Systems and Complexity in Health. New York: Springer New York; 2013.

76. Paley J, Eva G. Complexity theory as an approach to explanation in healthcare: a critical discussion. Int J Nurs Stud. 2011;48:269-79.

77. Byrne DS. Complexity Theory and the Social Sciences: An Introduction. London. New York: Routledge; 1998.

78. Estabrooks CA, Derksen L, Winther C, Lavis JN, Scott SD, Wallin L, ProfettoMcGrath J. The intellectual structure and substance of the knowledge utilization field: A longitudinal author co-citation analysis, 1945 to 2004. Implement Sci. 2008;3:49.

79. Estabrooks CA, Thompson DS, Lovely JJE, Hofmeyer A. A guide to knowledge translation theory. J Contin Educ Health Prof. 2006;26:25-36.

80. Crabtree BF, Nutting PA, Miller WL, McDaniel RR, Stange KC, Roberto JC, Stewart E. Primary care practice transformation is hard work: insights from a 15-year developmental program of research. Med Care. 2011;49:S28-35.

81. Cohen D, McDaniel RR, Crabtree BF, Ruhe MC, Weyer SM, Tallia A, Miller WL, Goodwin MA, Nutting P, Solberg LI, Zyzanski SJ, Jaén CR, Gilchrist V, Stange KC. A practice change model for quality improvement in primary care practice. J Healthc Manag Am Coll Healthc Exec. 2004;49:155-68. discussion 169-170.

82. Goodwin MA, Zyzanski SJ, Zronek S, Ruhe M, Weyer SM, Konrad N, Esola D Stange KC. A clinical trial of tailored office systems for preventive service delivery. The Study to Enhance Prevention by Understanding Practice (STEPUP). Am J Prev Med. 2001;21:20-8.

83. Stange KC, Goodwin MA, Zyzanski SJ, Dietrich AJ. Sustainability of a practice-individualized preventive service delivery intervention. Am J Prev Med. 2003:25:296-300

84. Tallia AF, Lanham HJ, McDaniel RR, Crabtree BF. 7 characteristics of successful work relationships. Fam Pract Manag. 2006;13:47-50.

85. Aita V, Mcllvain H, Susman J, Crabtree B. Using metaphor as a qualitative analytic approach to understand complexity in primary care research. Qual Health Res. 2003;13:1419-31.

86. Leykum LK, Lanham HJ, Pugh JA, Parchman M, Anderson RA, Crabtree BF, Nutting PA, Miller WL, Stange KC, McDaniel RR. Manifestations and implications of uncertainty for improving healthcare systems: an analysis of observational and interventional studies grounded in complexity science. Implement Sci. 2014;9:165.

87. Kernick D. Wanted-new methodologies for health service research. Is complexity theory the answer? Fam Pract. 2006;23:385-90.

88. Richardson KA, Midgley G. Systems theory and complexity: Part 4-The evolution of systems thinking. EMERGENCE-MAHWAH-LAWRENCE ERLBAUM-. 2007:9:166.

89. Phelan SE. A Note on the Correspondence Between Complexity and Systems Theory. Syst Pract Action Res. 1999;12:237-46.

90. Davis B, Sumara DJ: Complexity and Education: Inquiries into Learning, Teaching, and Research. Psychology Press; 2006

91. Granovetter M. The strength of weak ties: A network theory revisited. Sociol Theory. 1983;1:201-33.

92. Kadushin C. Understanding Social Networks: Theories, Concepts, and Findings. USA: Oxford University Press; 2012.

93. Kernick D. Complexity and Healthcare Organization: A View from the Street. Oxford: Radcliffe Publishing; 2004.

94. Daudt HML, van Mossel C, Scott SJ. Enhancing the scoping study methodology: a large, inter-professional team's experience with Arksey and O'Malley's framework. BMC Med Res Methodol. 2013;13:48.

95. Colquhoun HL, Letts LJ, Law MC, MacDermid JC, Missiuna CA. A Scoping Review of the Use of Theory in Studies of Knowledge Translation. Can J Occup Ther. 2010;77:270-9.

96. Garg AX, Hackam D, Tonelli M. Systematic Review and Meta-analysis: When One Study Is Just not Enough. Clin J Am Soc Nephrol. 2008:3:253-60.

\section{Submit your next manuscript to BioMed Central and we will help you at every step:}

- We accept pre-submission inquiries

- Our selector tool helps you to find the most relevant journal

- We provide round the clock customer support

- Convenient online submission

- Thorough peer review

- Inclusion in PubMed and all major indexing services

- Maximum visibility for your research

Submit your manuscript at www.biomedcentral.com/submit
Biomed Central 